\title{
miR-200c as a Predictive Biomarker for 5-Fluorouracil Chemosensitivity in Colorectal Cancer
}

\author{
Fatemeh Karimi Dermani ${ }^{1} \cdot$ Rezvan Najafi $^{1}$ (D) \\ Published online: 7 December 2017 \\ (C) Springer Science+Business Media, LLC, part of Springer Nature 2017
}

Colorectal cancer (CRC) is the third most common malignancy and the second leading cause of cancer-related mortality worldwide [1]. 5-Fluorouracil (5-FU) is generally administered for treating advanced colorectal cancer. However, the fact that some patients develop 5-FU resistance during the course of treatment is a major obstacle to effective therapy. Therefore, identifying the molecular mechanism is urgently needed to underlie the 5-FU resistance in CRC cells and develop novel diagnostic and prognostic biomarkers for 5-FU resistance that will improve the development of therapeutic strategies for CRC patients [2].

MicroRNAs are a set of newly discovered, non-coding small RNA molecules that target mRNA cleavage or translational repression through binding to the sequences on the $3^{\prime}$ untranslated region (3'UTR) of target mRNAs with complete or incomplete complementarity. MiRNAs significantly can act as oncogenes or tumor suppressors and play important roles in diverse biological processes of cancer, including tumor initiation, tumor progression, and chemoresistance [3]. A large number of miRNAs are considered to be dysregulated in CRCs, and some have involved in response to anti-cancer therapies through the regulation of drug metabolism, drug transport, DNA damage response, or cell apoptosis [4]. Prominently, a subset of miRNAs could act as non-invasive and potential predictive biomarkers in circulation for drug response. In this regard, several studies have shown that miR-200c is also involved in inhibiting the development of drug resistance in cancer cells [5]. However, the molecular mechanism as to how miR-200c modulates the chemosensitivity to 5-FU in colorectal cancer is poorly known.

Rezvan Najafi

re.najafi@umsha.ac.ir; najafi2535@gmail.com

1 Research Center for Molecular Medicine, Hamadan University of Medical Sciences, Hamadan, Iran
The evidence suggests that inhibition of miR-200c expression results in cancer cell resistance to chemotherapeutic drugs [6]. Studies have shown that miR-200c can potentially sensitize colorectal cancer cells to 5-FU and serves by inhibiting Bcl-2 expression and inducing apoptotic susceptibility to 5FU [7].

Toden et al. demonstrated that curcumin chemosensitizes CRC cells to 5-FU via the upregulation of miR-200c in 5fluorouracil-resistant (5FUR) cancer cell lines [8].

miR-200c inhibits epithelial-to-mesenchymal transition (EMT), proliferation, metastasis, and resistance to chemotherapeutic drugs and is also significant in the development of drug resistance of cancer cells $[9,10]$.

Tanaka et al. have suggested that the downregulated expression of miR-200c plays a role in selective resistance to chemotherapeutic drugs and EMT in CRC cells during repeated treatments with chemotherapeutic drugs [11].

Our recent study has shown that the inhibition of miR-200c correlated with the acquired resistance of colorectal cancer cells (HCT-116) to 5-FU. We investigated the effect of miR200c inhibition on apoptosis and the expression of PTEN and E-cadherin protein. Our results showed that LNA-anti-miR200c suppressed the expression of miR-200c, PTEN, and Ecadherin compared with the control cells. Furthermore, we observed that LNA-anti-miR-200c could promote cell proliferation through inhibition of caspase 3 activity and reduction of apoptosis that is mediated by PTEN expression [12].

In conclusion, we hypothesized that miR-200c could be candidate as a therapeutic tool for increasing 5-FU chemosensitivity in CRC. Thus, if the results confirm our hypothesis, miR-200c might be a possible biomarker to predict the response of colorectal cancer to 5-FU-based chemotherapy.

\section{Compliance with Ethical Standards}

Conflict of Interest The authors declare that they have no conflict of interest. 


\section{References}

1. Ranjbarnejad T, Saidijam M, Tafakh Ms, Pourjafar M, Talebzadeh F, Najafi R. Garcinol exhibits anti-proliferative activities by targeting microsomal prostaglandin E synthase-1 in human colon cancer cells. Hum Exp Toxicol. 2016:0960327116660865.

2. Li T, Gao F, Zhang X-P. miR-203 enhances chemosensitivity to 5fluorouracil by targeting thymidylate synthase in colorectal cancer. Oncol Rep. 2015;33(2):607-14. https://doi.org/10.3892/or.2014. 3646.

3. Xie T, Huang M, Wang Y, Wang L, Chen C, Chu X. MicroRNAs as regulators, biomarkers and therapeutic targets in the drug resistance of colorectal cancer. Cell Physiol Biochem. 2016;40(1-2):62-76. https://doi.org/10.1159/000452525.

4. Zheng T, Wang J, Chen X, Liu L. Role of microRNA in anticancer drug resistance. Int J Cancer. 2010;126(1):2-10. https://doi.org/10. 1002/ijc.24782.

5. Feng X, Wang Z, Fillmore R, Xi Y. MiR-200, a new star miRNA in human cancer. Cancer Lett. 2014;344(2):166-73. https://doi.org/ 10.1016/j.canlet.2013.11.004.

6. Chen Y, Sun Y, Chen L, Xu X, Zhang X, Wang B, et al. miRNA$200 \mathrm{c}$ increases the sensitivity of breast cancer cells to doxorubicin through the suppression of E-cadherin-mediated PTEN/Akt signaling. Mol Med Rep. 2013;7(5):1579-84. https://doi.org/10.3892/ mmr.2013.1403.
7. Qian X, Howell P, Li X, Qin X, Gao L, Xi Y. MiR-200c sensitizes colorectal cancer cells to 5-FU through Bcl-2-involved apoptotic pathway. Cancer Res. 2010;70(8 Supplement):4042. https://doi. org/10.1158/1538-7445.AM10-4042.

8. Toden S, Okugawa Y, Jascur T, Wodarz D, Komarova NL, Buhrmann C, et al. Curcumin mediates chemosensitization to 5fluorouracil through miRNA-induced suppression of epithelial-tomesenchymal transition in chemoresistant colorectal cancer. Carcinogenesis. 2015;36(3):355-67. https://doi.org/10.1093/ carcin/bgv006.

9. Kaboli PJ, Rahmat A, Ismail P, Ling K-H. MicroRNA-based therapy and breast cancer: a comprehensive review of novel therapeutic strategies from diagnosis to treatment. Pharmacol Res. 2015;97: 104-21. https://doi.org/10.1016/j.phrs.2015.04.015.

10. Karimi Dermani F, Saidijam M, Amini R, Mahdavinezhad A, Heydari K, Najafi R. Resveratrol inhibits proliferation, invasion, and epithelial-mesenchymal transition by increasing miR-200c expression in HCT-116 colorectal cancer cells. J Cell Biochem. 2017;118(6):1547-55. https://doi.org/10.1002/jcb.25816.

11. Tanaka S, Hosokawa M, Yonezawa T, Hayashi W, Ueda K, Iwakawa S. Induction of epithelial-mesenchymal transition and down-regulation of miR-200c and miR-141 in oxaliplatinresistant colorectal cancer cells. Biol Pharm Bull 2014 (0).

12. Heydari K, Saidijam M, Reza Sharifi M, Asl SS, Shabab N, Najafi R. The effect of miR-200c inhibition on chemosensitivity (5fluorouracil) in colorectal cancer. Pathol Oncol Res. 2017:1-7. 